\title{
Comparative Classification Efficiency of Self-organizing Map, Principal Component Analysis, and Hierarchical Cluster Analysis for Normal Dairy and Differently Pineapple Fortified Rasgulla
}

\author{
Tanmay Sarkar, Molla Salauddin, Sudipta Kumar Hazra and Runu Chakraborty*
}

\begin{abstract}
Rasgulla, the popular Indian dairy-based dessert, was fortified by using pineapple to improve its nutritional and different physical parameters like color, texture and sensory attributes. Being a highly nutritious, palatable fruit with excellent flavor and visual color characteristics, pineapple fortification provides an edge over normal dairy rasgulla. Comparative analysis of nutritional, $\mathrm{X}$-ray diffraction, texture and sensory characteristics for normal rasgulla sample and pineapple with different drying process (pulp (PP), convective (PH), microwave (PMW), microwave convective (PMWC), and freeze-dried (PF)) fortified rasgulla samples were done. $\mathrm{PH}$ was found with maximum carbohydrate $(40.60 \pm 2.04)$ and minimum protein content (5.91 \pm 0.95$)$. PMWC was found with the maximum total color difference $(\Delta E=48.79 \pm 3.33)$. Pineapple fortification did not alter the crystal characteristics of the rasgulla samples. Normal rasgulla showed the lowest hardness value while PMWC was the hardest one. The results obtained from the analysis were further comparatively segregated with the help of principal component analysis, hierarchical cluster analysis and self-organizing map to optimize the analysis frequency towards a more accurate result. It was found that more or less the same trends in results were obtained by all three analysis tools. It can be concluded that machine learning-based self-organizing map can be more sophistically used for classification between different parameters even in case of complex non-linear problems also. Among the entire samples microwave, convective dried pineapple rasgullawas found to be the best in terms of all the observed parameters.
\end{abstract}

Keywords: Machine learning; Food fortification; Dairy; Neural network; Nutrition; Clustering.

Received: 10 August 2020; Accepted date: 30 September 2020.

Article type: Research Article .

\section{Introduction}

Rasgulla or sweetened cheese ball is a very popular dairybased dessert in all over India. Fortification is one kind of approach to improve the nutritional and physical attributes (sensory, color, texture) of rasgulla, which makes it more attractive and healthy. Pineapple is a tropical seasonal fruit that is well known for its excellent nutritional characteristics and can be used as a fortifying agent in rasgulla. Pineapple is rich in beta carotene, which results in excellent yellowish color. Due to fortification, yellow color is transferred into normal rasgulla and makes it more attractive. ${ }^{[1]}$ Pineapple is a rich source of minerals like phosphorus, zinc, and manganese;

\footnotetext{
Department of Food Technology and Biochemical Engineering, Faculty of Engineering and Technology, Jadavpur University, Jadavpur, Kolkata 700032, India.

*E-mail: crunu@hotmail.com (R. Chakraborty)
}

phytochemicals like coumarins, flavonoids, and phenols; and vitamins like ascorbic acid, and vitamin $\mathrm{B}_{6}$. Pineapple is available only from June to November, which is the main concern with pineapple industry. Dehydration may be the option to overcome the problem and the dehydrated pineapple can be in use over the year. From previous studies, it has been substantiated that pineapple fortification improves the textural, sensory and color attributes of dairy products. ${ }^{[1]}$ Also due to pineapple fortification, the nutritional qualities of rasgulla get enriched.

Other physical attributes of rasgulla like sensory and texture were also improved due to pineapple fortification. ${ }^{[1]}$ By X-ray diffraction (XRD) analysis, more information was obtained about the effects of pineapple fortification on sensory qualities like crystal formation. For comparative studies, four types of dried pineapple pulp (convective $(\mathrm{PH})$, microwave (MR), microwave convective (MCR) and freeze (PF) dried) along with raw pineapple pulp were used for fortification. The 
mathematical model design of artificial neural network (ANN) was enlivened by the bio-inspired algorithm or network and the model consisting of basic processing units, which was worked out by following a leaning methodology to store scientific knowledge. Among several types, self-organizing maps (SOM), which are often represented as SOM or denoted as per the name of "Kohonen Map", is a kind of artificial neural network (ANN) that works through unsupervised learning. ${ }^{[2]}$ Application of SOM was reported for the classification and identification of vegetable oils. ${ }^{[3-5]}$ Multivariate data principal component analysis (PCA) generally was followed for analyses. To mitigate the classification and clustering problems more accurately, SOM can be used for multivariate data analysis. SOM is constructed through a data matrix, and arranged spatially following a specific kind of definite topology, commonly a twodimensional hexagonal or rectangular grid. The input space and the weight vector share the same dimension. ${ }^{[6-9]}$ Through the relevancy of metric relations and topological representation obtained from the data, SOM constricts the data. For its non-linear characteristic, SOM could be extensively used even for complex problems also. Dimension reduction is generally conducted with PCA, whereas SOM is generally used for clustering purposes. In food processing industries, SOM is not in practice widely, though it is a very efficient clustering tool and may have immense importance in the field of food-related research with a large amount of data set.

This study aims to investigate about the effects of pineapple fortification on the nutritional, textural, color and sensory profile of normal dairy rasgulla. Apart from this, the main aim of the study is to classify normal dairy rasgulla and differently fortified rasgulla based on their nutritional, textural, color and sensory attributes through linear PCA and HCA method along with non-linear machine learning process of SOM.

\section{Experimental section}

\subsection{Materials and methods}

The pineapple was procured from local market of Kolkata, India. The quality and ripeness of the procured materials were ascertained by physical verification. Milk was procured from HACCP and ISO 9622:2013 certified dairy industry. The pineapple drying was conducted per our previous work. ${ }^{[10]}$ Normal rasgulla (NR), fresh pulp (PP), convective (PH), microwave (PMW), microwave convective (PMWC) and freeze-dried (PF) pineapple fortified rasgulla were processed as per our previous work. ${ }^{[10]}$

\subsection{Nutritional analysis}

By following AOAC 2000, crude protein content and moisture content (MC) of the normal dairy and pineapple fortified rasgullas were estimated ${ }^{[11]}$ By following Sarkar et al., 2020 carbohydrate, fat and ash content were measured. ${ }^{[12]}$

\subsection{Texture and color analysis}

$\mathrm{L}$ or lightness, $\mathrm{b}$ or yellow-blue and a or green-red values, $\Delta \mathrm{E}$ or the net difference in color, hue and chroma were estimated following by Granato et al., 2010. ${ }^{[13]}$ The analysis of textural parameters was accompanied by Omolola et al., 2015 process with the P5 probe and the TA.HD Plus Texture Analyzer, USA. ${ }^{[14]}$ The probe was allowed to penetrate at a speed of 25 $\mathrm{mm} / \mathrm{s}$ and the sample was placed over stainless steel (SS-305) platform with a hole at the center $(1.25 \mathrm{~cm}$ diameter $)$. The rasgulla sample was placed over the platform, the centroid of rasgulla, center of the hole and the center of the probe was aligned at $180^{\circ}$ vertical straight line.

$$
\begin{aligned}
& \Delta E=\sqrt{\left(L_{N R}-L_{P R}\right)^{2}+\left(a_{N R}-a_{P R}\right)^{2}+\left(b_{N R}-b_{P R}\right)^{2}} \\
& \text { Chroma }=\sqrt{a_{R}^{2}+b_{R}^{2}} \\
& \text { Hue angle }=\tan ^{-1} \frac{b_{R}}{a_{R}} \\
& \text { Browning Index }(I B)=\frac{100 \times(x-0.31)}{0.17} \\
& x=\left(a+1.75 L_{P R}\right) /\left(5.645 L_{P R}+a_{P R}-3.012 b_{P R}\right)
\end{aligned}
$$

where, $\mathrm{L}_{\mathrm{NR}}, \mathrm{b}_{\mathrm{NR}}, \mathrm{a}_{\mathrm{NR}}, \mathrm{L}_{\mathrm{PR}}, \mathrm{b}_{\mathrm{PR}}$ and $\mathrm{a}_{\mathrm{PR}}$ stands for the value of lightness, yellow-blue and green-red values for normal rasgulla and pineapple rasgullas respectively.

\subsection{Analysis of sensory attributes}

For sensory analysis, a 30-member (19 male and 11 female members in the age group of 22-58 years) semi-trained panel (the entire panelists were regular consumers of rasgulla) of the institute was formed and the evaluation was carried out in the laboratory itself. Color, flavor, texture, mouthfeel, overall acceptability, and after taste were assigned as per our previous work. ${ }^{[10]}$ The entire panel was chosen in a manner that they were familiar with both the rasgulla and pineapple. Panelists were given $15 \mathrm{~g}$ of each sample (the samples were supplied thrice and the mean score was considered) with a randomly generated three-digit code. The seating arrangement for each panelist was separated with wooden partitions, between two successive consumptions by the panel members rinsed their mouth with potable water and took extruded rice for neutralisation of taste buds.

\subsection{X-ray diffraction (XRD)}

By using the Rigaku MiNiflex diffractometer, XRD behaviors of entire rasgulla samples (normal dairy and fortified ones) were carried out. A quartz slide was prepared to place rasgulla samples with a $0.5 \times 100 \times 50 \mathrm{~mm}$ grove in the middle. The input current was set at $30 \mathrm{~mA}$, while the potential difference was $35 \mathrm{kV}$ and the wavelength was $0.15 \mathrm{~nm} .0 .02^{\circ}$ of step angle was maintained while the rate of the scan was 1 second for each step and the diffraction angle was varied from $5^{\circ}$ to $50^{\circ} \cdot[12]$

\subsection{Data analysis}


One-way analysis of variance (ANOVA) followed by Tukey's test was performed considering $95 \%$ confidence level. All the data were taken as the average of the discrete experimental analysis performed in triplicate. Correlation analysis, principal component analysis (PCA) and hierarchical cluster analysis (HCA) were carried out with R Studio software version 3.4.4 (2018-03-15). The SOM operations were performed based on the selected $6 \times 6$ neuron layers. MATLAB R2014b (Math Works Inc., USA) was the software selected to conduct the SOM. Fig. 1 represents the SOM model constructed for data analysis.

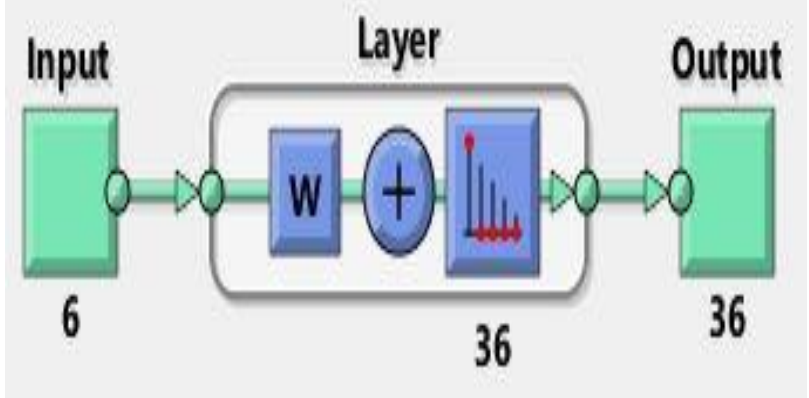

Fig. 1 Representative model for shelf organizing map (SOM), which consist of six input and thirty-six numbers of neurons.

\section{Results and discussion}

3.1Nutritional composition

The proximate composition of all the rasgulla samples was reported in Table 1. From Table 1, it has been clear that the amount of moisture present in NR has been significantly $(\mathrm{p}<$ 0.05 ) lower than the amount of moisture present in the pineapple fortified rasgulla samples. But PP and rasgulla fortified with differently dried pineapple were statistically insignificantly varied in terms of moisture content. This might be due to the incorporation of relatively higher moisture from pineapple fruit pulp $(80.78 \pm 3.2 \%)$ compared to chhana $(51.70-53.41 \%)$, which is the raw material for rasgulla. All the pineapple fortified rasgulla samples absorbed more or less similar amount of sugar syrup during soaking, which may be the reason behind the insignificant difference in the moisture content for differently fortified rasgullas.

Ash content of normal rasgulla was varied in a range of $0.75 \pm 0.005-1.04 \pm 0.03 \%$ as reported by different literature and in this study, the ash content of NR was found $1.19 \pm 0.03 \% .^{[15-16]}$ The pineapple was reported to have an ash content as $(0.45 \pm 0.002) \%$, which might differ with respect to different cultivar. ${ }^{[17]}$ Insignificant changes $(p>0.05)$ were observed in the ash content of all the pineapple fortified rasgulla samples as minerals remained unaffected by any kind of drying treatment. ${ }^{[18]}$

Similar to the case of fat content, insignificant changes were observed between all the pineapple fortified rasgulla samples though NR was found to have a significantly higher amount of fat content due to the high amount of channa in it. NR and pineapple fruit was reported to have a fat content $2.72 \pm 0.10-14.41 \pm 6.43 \%$ and $0.956 \%$, respectively. ${ }^{[15-17]}$

Table 1 Nutritional composition of normal and differently fortified rasgulla

\begin{tabular}{|c|c|c|c|c|c|c|}
\hline Sample & $\operatorname{MC}(\%)$ & Ash (\%) & Protein $(\%)$ & Fat $(\%)$ & $\begin{array}{c}\text { Carbohydrat } \\
\text { e }(\%)\end{array}$ & Total solids (\%) \\
\hline NR & $44.23 \pm 1.56^{\mathrm{b}}$ & $1.01 \pm 0.05^{\mathrm{b}}$ & $8.73 \pm 0.80^{\mathrm{a}}$ & $8.01 \pm 0.79^{\mathrm{a}}$ & $38.02 \pm 1.15^{\mathrm{c}}$ & $52.91 \pm 2.36^{\mathrm{a}}$ \\
\hline PP & $45.45 \pm 1.09^{\mathrm{a}}$ & $1.03 \pm 0.01^{\mathrm{b}}$ & $7.32 \pm 0.89^{\mathrm{b}}$ & $6.58 \pm 0.81^{\mathrm{b}}$ & $39.62 \pm 1.08^{\mathrm{b}}$ & $42.33 \pm 1.07^{\mathrm{b}}$ \\
\hline $\mathrm{PF}$ & $45.39 \pm 1.60^{\mathrm{a}}$ & $1.03 \pm 0.03^{\mathrm{b}}$ & $7.33 \pm 0.21^{\mathrm{b}}$ & $6.04 \pm 0.57^{c}$ & $40.21 \pm 0.96^{\mathrm{a}}$ & $54.29 \pm 1.44^{\mathrm{a}}$ \\
\hline $\mathrm{PH}$ & $45.11 \pm 1.72^{\mathrm{a}}$ & $1.08 \pm 0.09^{\mathrm{b}}$ & $7.30 \pm 0.85^{\mathrm{b}}$ & $5.91 \pm 0.95^{\mathrm{c}}$ & $40.60 \pm 2.04^{\mathrm{a}}$ & $52.26 \pm 1.87^{\mathrm{a}}$ \\
\hline PMW & $45.85 \pm 1.12^{\mathrm{a}}$ & $1.12 \pm 0.15^{\mathrm{a}}$ & $7.22 \pm 0.49^{c}$ & $6.09 \pm 0.58^{\mathrm{c}}$ & $39.72 \pm 1.19^{\mathrm{a}}$ & $52.92 \pm 2.05^{\mathrm{a}}$ \\
\hline PMWC & $45.40 \pm 1.33^{\mathrm{a}}$ & $1.09 \pm 0.01^{\mathrm{b}}$ & $7.34 \pm 0.75^{b}$ & $6.18 \pm 0.77^{c}$ & $40.03 \pm 1.20^{\mathrm{a}}$ & $53.88 \pm 1.96^{\mathrm{a}}$ \\
\hline
\end{tabular}

[Results are expressed as mean \pm standard deviation. Where, NR-normal rasgulla, PP-pulp rasgulla, PH-hot air, PF-freeze, PMWmicrowave, PMWC-microwave convective dried pineapple fortified rasgulla. MC- moisture content. Same superscripted letters in a column represented the insignificant difference]. 
The protein contents of all the pineapple fortified rasgulla samples were significantly decreased with respect to NR and this was due to different drying processes, which caused severe damages to the hydrogen bond and different hydrophobic interactions in the protein structure. This resulted in the denaturation of the protein. Hassan et al., 2007 reported a decrease in protein content of hot air dried Capparaceae compared to fresh; ${ }^{[19]}$ Amezquita et al., 2018 reported a higher amount of protein content in freeze dried pear-peel compared to hot air drying. ${ }^{[20]} \mathrm{NR}$ and pineapple fruit were reported to have a protein content $(3.91 \pm 0.12-8.24 \pm 0.05) \%$ and $(1.19 \pm 0.03) \%$ respectively. ${ }^{[15-17]}$

The Carbohydrate content of NR $(37.02 \pm 1.03 \%)$ was found higher than PP $(29.616 \pm 1.12 \%)$ due to the higher carbohydrate content of NR than raw pineapple fruit pulp. But in the case of differently dried pineapple fortified rasgulla samples, carbohydrate content was found significantly higher than PP and NR as well. This phenomenon occurred due to the different drying treatment which caused an increase in the dry matter content of pineapple thus caused a higher carbohydrate amount in all dried pineapple pulp. Ajayi et al., 2017 reported an increase in carbohydrate content of microwave and oven dried ginger than raw ginger sample. ${ }^{[21]} \mathrm{NR}$ and pineapple fruits were reported to have a protein content $(36.57 \pm 0.51$ $50.74 \pm 0.49) \%$ and $(13.04 \pm 2.03) \%$ respectively. ${ }^{[15-17]}$ The total solid content of all the dried pineapple fortified rasgulla samples were significantly $(p<0.05)$ increased compared to $\mathrm{PP}$ due to the removal of moisture from pineapple fruit pulp.

\subsection{Color}

To enhance any product's aesthetic appeal towards consumer acceptability, color is primarily one of the key parameters. One of the prime objectives of pineapple fortification of normal rasgulla is to incorporate pineapple's characteristic yellow color in normal rasgulla. The major color pigments present in pineapple were carotenoids especially $\beta$ - carotene and xanthophyll, which were well known as one of the major natural color pigment groups. ${ }^{[1]}$ The total color difference in all the pineapple fortified rasgulla samples is found in the following order $-\mathrm{PMWC}(\Delta \mathrm{E}=48.79 \pm 3.33)>\mathrm{PMW}(\Delta \mathrm{E}=$ $15.9 \pm 1.99)>\mathrm{PH}(\Delta \mathrm{E}=8.62 \pm 1.03)>\mathrm{PF}(\Delta \mathrm{E}=7.41 \pm 0.93)>$ $\mathrm{PP}(\Delta \mathrm{E}=6.26 \pm 0.89)$. The development of color in pineapple fortified rasgulla might be due to the color incorporation through carotenoid pigment from pineapple, non-enzymatic browning reaction during different drying processes of pineapple, Maillard reaction during cooking of different rasgulla in sugar syrup. Bandyopadhyay et al., 2008 also reported the development of color by using carrot in normal rasgulla. ${ }^{[23]}$ Maillard reaction, the characteristic reaction for non-enzymatic browning process, is referred to a group of reactions that follows a very complex reaction pathway starting with the reaction of aldose sugars and amino acid of casein and then following by amadori arrangements. It further underwent through several pathways, all of which finally resulted in the formation of melanoidins, a nitrogenous colored polymer. ${ }^{[23]}$ Several studies reported that the rate of Maillard reaction was increased with an increase in temperature. ${ }^{[24]}$ It was seen that the color difference was significantly increased with respect to different drying treatments and the highest color difference was observed in the case of PMWC followed by PMW, PH, and PF. Izli et al., 2018 reported a similar trend of color difference in the case of microwave, convective and freeze-dried pineapple slices compared to fresh pineapple slices. ${ }^{[25]}$ Ramallo et al., 2012 also reported an increase in the yellowish color of pineapple in the convective drying process. ${ }^{[26]}$ Pham et al., 2018 also reported a higher change in color values in microwave convective drying compared to convective dried kiwi fruit. ${ }^{[2]}$ Higher temperature drying treatment such as PMWC and PMW caused an increase in total carotene content compared to mild and low drying treatment such as $\mathrm{PH}$ and $\mathrm{PF}$. This might be due to the better extraction of carotene at higher temperature treatment that was also considered as an important reason for the color difference trend in pineapple fortified rasgulla. Also, the presence of lysine in channa in a higher amount favored the browning reaction rate during the boiling of rasgulla into sugar syrup. ${ }^{[28]}$

The color difference in pineapple fortified rasgulla might be due to the browning reaction and the Maillard reaction during the drying and cooking process. With browning, the ' $L$ ' values of the pineapple fortified samples were decreased, resulting in a decrease in the denominator of IB, leading to an increase in the IB values. A significantly higher IB was observed for PH, PMW and PMWC compared to NR. Similar to the trend of total color difference, IB showed the highest value for PMWC followed by PMW, PH and PF.

\subsection{Texture}

The texture is considered as one of the most important quality parameters that is determined through the values of a set of parameters like chewiness, adhesiveness, gumminess, hardness, cohesiveness, springiness and stiffness obtained from texture profile analysis. There are several studies on the texture of rasgulla with respect to different characteristics and other conditions as well. ${ }^{[29-33]}$

Hardness values (expressed in Newton $(\mathrm{N})$ ) of all the rasgulla samples were observed in the following order $-\mathrm{NR}$ $(5.26 \pm 0.29 \mathrm{~N})<\mathrm{PP}(5.31 \pm 0.19 \mathrm{~N})<\mathrm{PF}(5.38 \pm 0.27 \mathrm{~N})<\mathrm{PH}$ $(5.41 \pm 0.23 \mathrm{~N})<\mathrm{PMW}(5.49 \pm 0.37 \mathrm{~N})<\operatorname{PMWC}(5.55 \pm 0.49 \mathrm{~N})$. From all these values, it can be seen that as the fat content of rasgulla samples (Table 1) is decreased as the hardness value is increased. Chavan et al., 2011 also reported a similar trend of hardness increasing with a decrease in fat content. ${ }^{[29]} \mathrm{Also}$, integrity in protein structure has an important factor for the hardness of rasgulla. ${ }^{[30-32]}$ It was observed that the hardness of rasgulla decreased as the degree of denaturation of protein was decreased, or the protein content was increased. Also, an increase in the total solid content might have a little bit effect on the hardness trend of all the rasgulla samples. ${ }^{[5]}$

An opposite trend was observed in the case of cohesiveness 
as compared to hardness. Cohesiveness values of all the rasgulla samples were found as the following order $-\mathrm{NR}$ $(0.66 \pm 0.09)>\mathrm{PP}(0.59 \pm 0.08)>\mathrm{PF}(0.55 \pm 0.01)>\mathrm{PH}$ $(0.51 \pm 0.03)>\operatorname{PMWC}(0.49 \pm 0.02)>$ PMW $(0.47 \pm 0.01)$ Kumar et al., 2003 reported that an increase in fat content resulted in a decrease in the cohesiveness value of all the rasgulla samples and this observation was consistent with our study. ${ }^{[30]}$ Fat dispersion and protein matrix may influence the cohesiveness of all the rasgulla samples. ${ }^{[32]}$

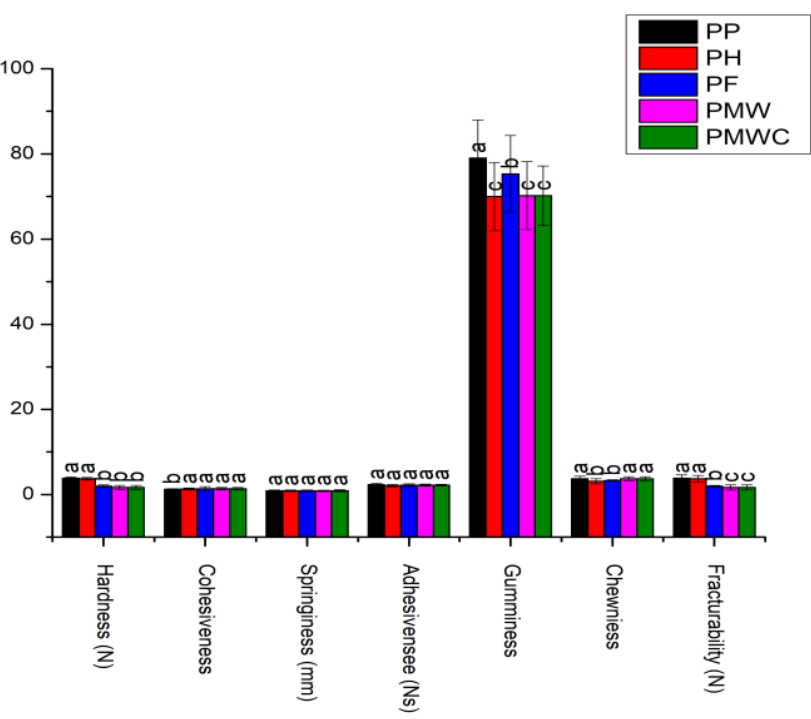

Fig. 2 Texture parameters for different rasgulla samples [Where, NR-Normal rasgulla, PH-Hot air dried pineapple rasgulla, PFFreeze dried [pineapple rasgulla, PMW-Microwave dried pineapple rasgulla, PMWC-Microwave convective dried pineapple rasgulla].

Springiness is considered as the most vital parameter for the texture of rasgulla. In this study, springiness values of all the rasgulla samples were found as follows - PH $(5.91 \pm 0.55$ $\mathrm{mm})>\mathrm{PMW}(5.89 \pm 0.44 \mathrm{~mm})>\operatorname{PMWC}(5.87 \pm 0.41 \mathrm{~mm})>\mathrm{PF}$ $(5.84 \pm 0.69 \mathrm{~mm})>\mathrm{PP}(5.83 \pm 0.51 \mathrm{~mm})>\mathrm{NR}(5.81 \pm 0.58 \mathrm{~mm})$. It was observed that a higher fat content resulted in a lower springiness value, which is similar to the findings reported by Kumar et al., 2003. ${ }^{[29]}$

Adhesiveness of all the rasgulla samples were found as follows $-\mathrm{NR}(0.093 \pm 0.003 \mathrm{~N}-\mathrm{mm})>\mathrm{PP}(0.088 \pm 0.002 \mathrm{~N}-$ $\mathrm{mm})>\mathrm{PF}(0.085 \pm 0.002 \mathrm{~N}-\mathrm{mm})>\operatorname{PMWC}(0.082 \pm 0.001 \mathrm{~N}-$ $\mathrm{mm})>\operatorname{PMW}(0.081 \pm 0.002 \mathrm{~N}-\mathrm{mm})>\mathrm{PH}(0.078 \pm 0.000 \mathrm{~N}-$ $\mathrm{mm})$. In the case of adhesiveness, protein content was found as the most predominant factor. A higher protein content resulted in a higher adhesiveness value. ${ }^{[30]}$ The texture characteristics of different rasgulla samples were shown in Fig. 2. Gumminess was found to be the highest in PP followed by PF. PH, PMW and PMWC showed lower gumminess values and they were statistically insignificantly different $(\mathrm{p}>0.05)$.

\subsection{X-ray diffraction (XRD) analysis}

From the structural aspects point of view, XRD is very much helpful to get an idea about the crystalline -amorphous nature of any sample structure. In XRD, the presence of any crystal in the structure is validated by the characteristic peaks output in the diffractogram. On the other side, the absence of any characteristic peaks in the diffractogram indicates the amorphous nature of the product structure. The Diffractogram of all the samples were reported in Fig. 3. It is obvious from Fig. 3 that in all the samples, no observed sharp peak signifies their amorphous nature.

Lower molecular weight compounds like sugars (lactose, sucrose, fructose, glucose), and organic acids were mainly responsible for the formation of amorphous structure in all the rasgulla samples. In all the rasgulla samples, no crystal was formed due to two reasons - low molecular weight sugar in the rasgulla samples was unable to form any crystalline structure and Jaya et al., 2009 reported the same phenomena for normal yogurt, ${ }^{[35]}$ the rapid drying treatment of pineapple pulp in $\mathrm{PH}$, PMW, PMWC, which was insufficient to form any crystal. Crystal formation generally takes place at a temperature of more than $50{ }^{\circ} \mathrm{C}$. In freeze-drying, the lower drying temperature is also inhibited to form any crystalline structure in PF. In the case of PP, similar diffractometric characteristics in line PH, PMW, PMWC were observed. Low operating temperature $\left(<50{ }^{\circ} \mathrm{C}\right)$ and low lactose sugar concentration might inhibit any crystal formation in the case of NR and PP. All freeze dried rasgulla samples had shown a similar kind of diffractogram patterns though significant variation in their respective intensities was observed in Fig. 3. PMW, PMWC and $\mathrm{PH}$ resulted in higher intensities, and on the other side gentle drying treatment like FD resulted in a lower intensity. Prolonged drying time in hot air drying operation caused the absorption of higher energy that resulted in the higher intensity of the PH sample among all. Jaya et al. 2009 and Sengupta et al., 2017 reported similar results in the case of convective, microwave vacuum and freeze, drying of normal yogurt and Spirulina platensis enriched soy yogurt. ${ }^{[34-35]}$

\subsection{Sensory analysis}

In a new product development, sensory characteristics analysis is the last lap of the whole process which gives an instant feedback about the final product with respect to different parameters. In this study, sensory analysis of all the rasgulla samples were carried out by using a 0-9 point hedonic scale on total twelve parameters: color, mouthfeel, texture, flavor, chewiness, overall acceptability, visual appearance, sweetness, sourness, juiciness, bitterness, and aftertaste (Fig. 4).

For the color attribute, all the sensory scores were given based on the development of yellow color in fortified pineapple rasgulla samples and for flavor also all the sensory scores were given on the basis of the development of pineapple flavor in fortified pineapple rasgulla samples Color is considered as a very first impression of the consumer for the newly developed product and it was observed that PMWC got the highest score for color followed by PMW, PH, PF, and PP. In PMWC, the higher degree of incorporation of characteristic 


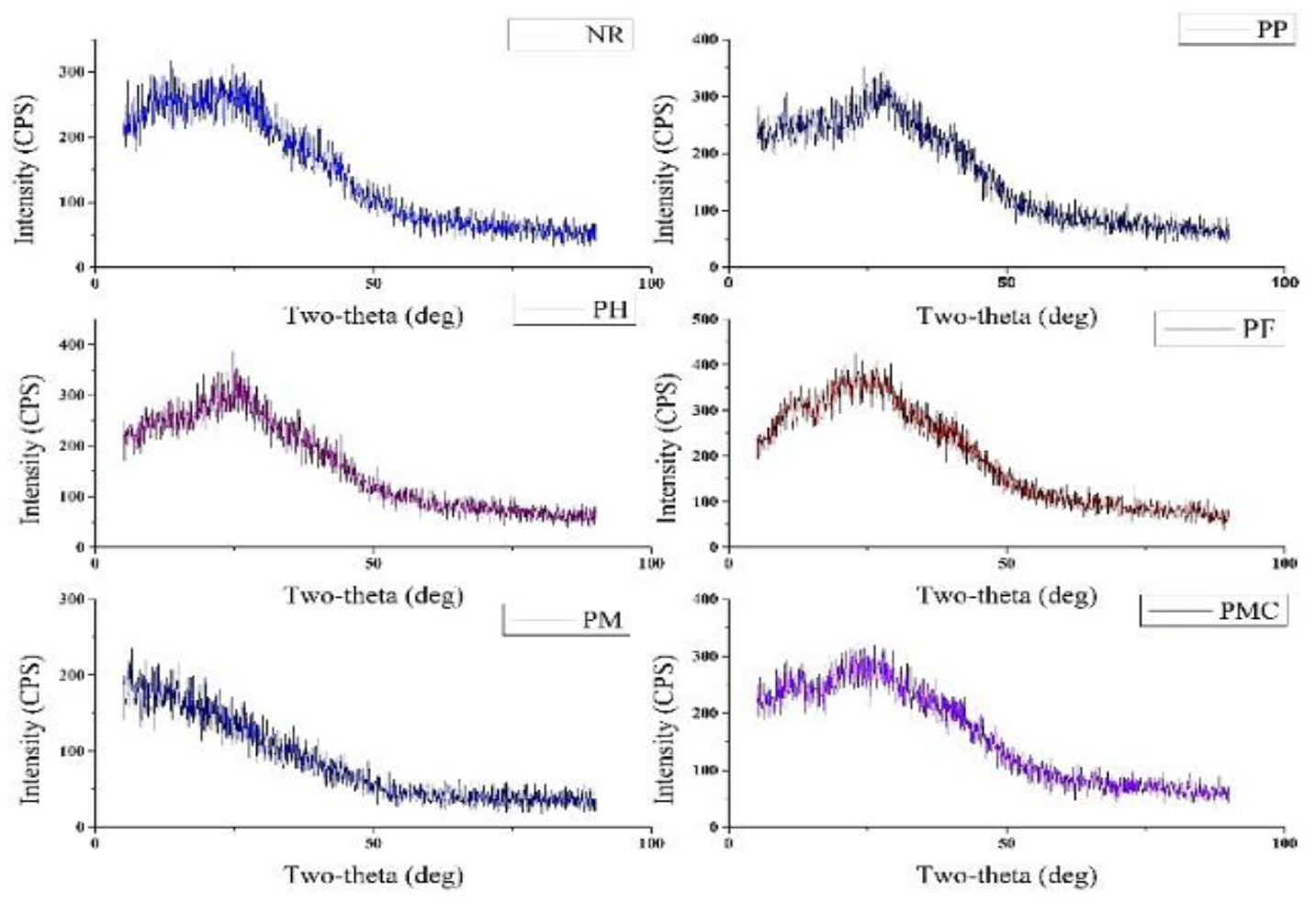

Fig. 3 XRD diffractogram of NR-normal rasgulla, PP-pulp rasgulla, PH-hot air, PF-freeze, PMW-microwave, and PMWC-microwave convective dried pineapple fortified rasgulla.

yellow color from pineapple led to the scored highest among all. Bandyopadhyay et al., 2008 reported color development in normal rasgulla by using carrot. ${ }^{[22]}$

On the other side, for mouthfeel and texture, NR was found with the highest score followed by the pineapple fortified rasgulla as the hardness values of all the pineapple fortified rasgulla samples were found higher than that of the NR. Among the fortified rasgulla samples, PP was found with the highest score for mouthfeel. While the PH, PF, PP were found with the highest scores for texture.

For chewiness, opposite result was observed where PMWC and $\mathrm{PH}$ were adjudged to get the highest scores followed by PP, PF, PMW and NR. On the basis of pineapple's characteristics, flavor incorporation in fortified rasgulla samples PP was adjudged to get the highest score followed by $\mathrm{PF}, \mathrm{PH}, \mathrm{PMW}$ and PMWC. Heat treatment might be a reason for the trend of flavor development in pineapple rasgulla samples. From an overall accessibility point of view, PH, PF and NR were scored highest followed by PP, PMW and PMWC. The PP, PMW and PMWC were insignificantly different in terms of flavor attributes.

\subsection{SOM, PCA and HCA analysis}

Definite integral form B ( $\beta$ ) of the local stimulation of a singledimensional neural field can be represented as follows.

$$
B(\beta)=\int_{0}^{\beta} \beta(s) d s
$$

The equation (6) evidenced that, for an input parameter with a non-zero value inconsistent with a specific active neural zone accepting maximum input of $s$ unit. $B$ is the width of the active neural zone.

SOM was proposed by Kohonen 1990, which was somewhat identical to Amari's self-arranging neural network though an array of 2D units. ${ }^{[2]} \mathrm{A}$ high-pitched auto on-off edgeways interconnected neural net of the proposed model was constructed, the specific unit with the highest stimulation emerged as the operating unit while the others were in the dormant stage. The unit weight $\mathrm{j}^{\text {th }}$ vector for learning is represented as follows.

$W_{v}=\left[y-\delta W_{j} c\right] \sum_{m} i_{j m} z_{m}$

where $m$ represents a constant value, which is a positive integer, $\sum_{m} i_{j m} z_{m}$ represents a regular adjacent function. $z_{m}$ represents neighbor neuron output activity, which depicts the winning capacity of unit mover unit $j$; $i_{j m}$ represents the distance function within neuron $\mathrm{j}$ and neuron $\mathrm{m}$. $-\delta W_{j} \sum_{s} W_{j s} y_{s}$ stands for steady-state maintenance term for adjacent neuron synaptic effect. The $W_{j}$, weight of $\mathrm{j}$ neuron is directly proportional to the noise or disturbance of the model itself. Model disturbance from $\mathrm{s}$ is intervened by the 


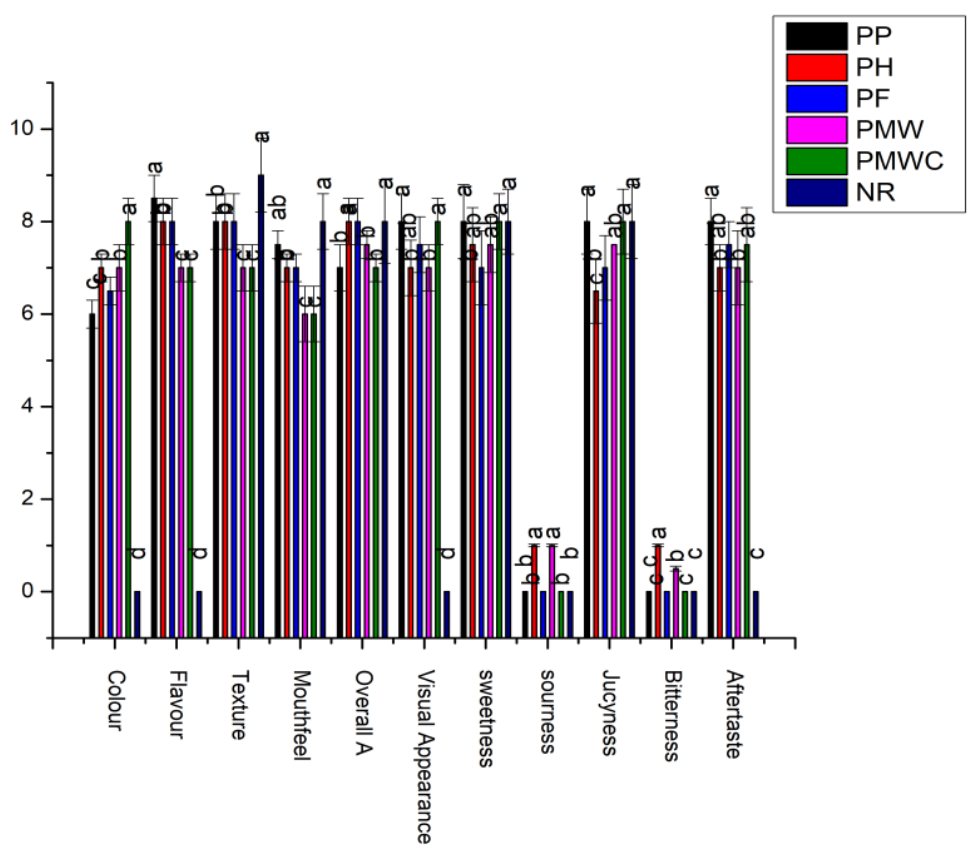

Fig. 4 Hedonic ratings of sensory scores for different rasgulla samples. [Where, NR-Normal rasgulla, PH-Hot air dried pineapple rasgulla, PF- Freeze dried [pineapple rasgulla, PMW-Microwave dried pineapple rasgulla, PMWC-Microwave convective dried pineapple rasgulla.]

potentiality of deferred synaptic effect and $W_{j s y s}$ is proportional to the forgetting effect, which can be modelled through $\sum_{s} W_{j s} y_{s}$ using the forgetting effect. We assumed that index s varied for all input parameter y and $\sum_{m} i_{j m} z_{m}=\beta$, where $\beta$ is a scalar component and positive quantity which does not depend on the values of $\mathrm{W}_{\mathrm{js}}$ and ys. Equation (7) can be represented on its vector form as follows. $W=\beta y-\gamma W\left[W^{U} y\right]$

To form a differential equation, Equation (8) is multiplied with $2 \mathrm{~W}^{\wedge} \mathrm{U}$, which ultimately resulted in Equation (9):

$2 W^{U} W=\frac{d\|W\|^{2}}{d u}=2 W^{U} y\left[\beta-\gamma\|W\|^{2}\right]$

Therefore, from SOM, it is anticipated that $W$ can be spontaneously normalized and does not depend on input parameter $\mathrm{y}, \mathrm{W}$ pivots within the $2 \mathrm{D}$ space and the directed in accordance with the mean value of input parameter (Fig. 2) for unvarying neighbor function.

From Fig. 8 principal component analysis of all the nutritional, textural and sensory parameters can be observed where all the parameters were divided along with twocomponent namely principal component 1 (PC 1) and principal component 2 (PC 2). These two components cumulatively contributed about $75.18 \%$ of the total variance. Among the six types of rasgulla samples - PMW, PH was found in the positive co-ordinate of PC 2; PMWC was found on the negative co-ordinate of $\mathrm{PC} 1$; PF was found on positive co-ordinate of PC 1; PP, NR was found on the negative coordinate of PC 2. Distribution arrangement of all the observed parameter (moisture content (MC), protein, carbohydrate, fat, ash, colour (C), total colour difference (Del E), texture (T), flavour (F), mouthfeel (MF), overall acceptability (OA), adhesiveness, springiness, chewiness, gumminess, hardness $(\mathrm{H})$, cohesiveness, chroma, L, a, b, hue, fracturability, visual appearance (VA), sweetness, sourness, juiciness, bitterness, aftertaste) of all the rasgulla samples were found in Fig. 8. Total six clusters were formed by all the parameters and those were - cluster 1 (springiness, gumminess, chewiness, H, VA, carbohydrate, Del E, aftertaste), cluster 2 (sourness, bitterness, b, chroma), cluster 3 (OA, L, hue, ash), cluster 4 (cohesiveness, fracturability, T, protein, fat, adhesiveness, MF), cluster 5 (sweetness, juiciness), cluster 6 (MC). From PCA model, PMW scored the highest $(0.4524)$ followed by PMWC (0.3822), PH (0.1452), PF (-0.1936), PP (-0.2825) and NR (0.5037). Therefore, from the PCA it can be concluded that PMW was the best sample in terms of the analyzed parameters followed by PMWC, PH, PF, PP and NR. 


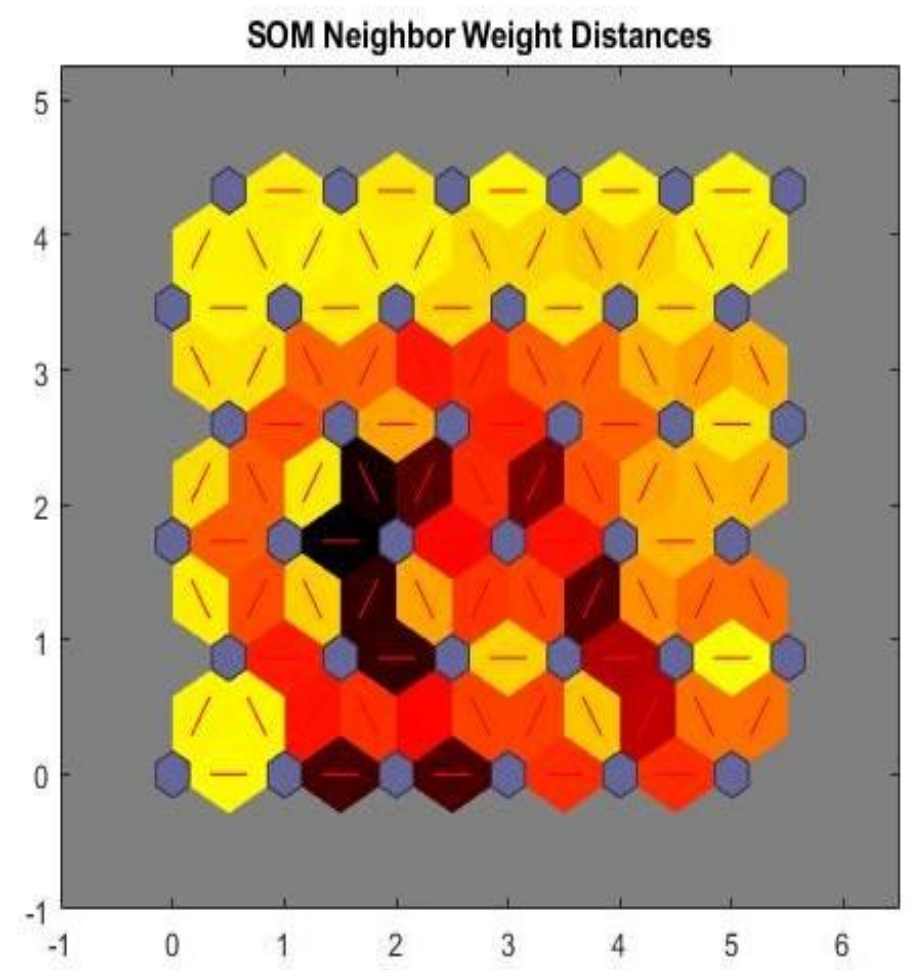

Fig. 5 Shelf organizing map (SOM) neighbor weight distance presenting nutritional, colour, texture and sensory attribute based cluster arrangements for normal rasgulla (NR) and differently fortified PH-Hot air dried pineapple rasgulla, PFFreeze dried pineapple rasgulla, PMW-Microwave dried pineapple rasgulla, PMWC- Microwave convective dried pineapple rasgulla.
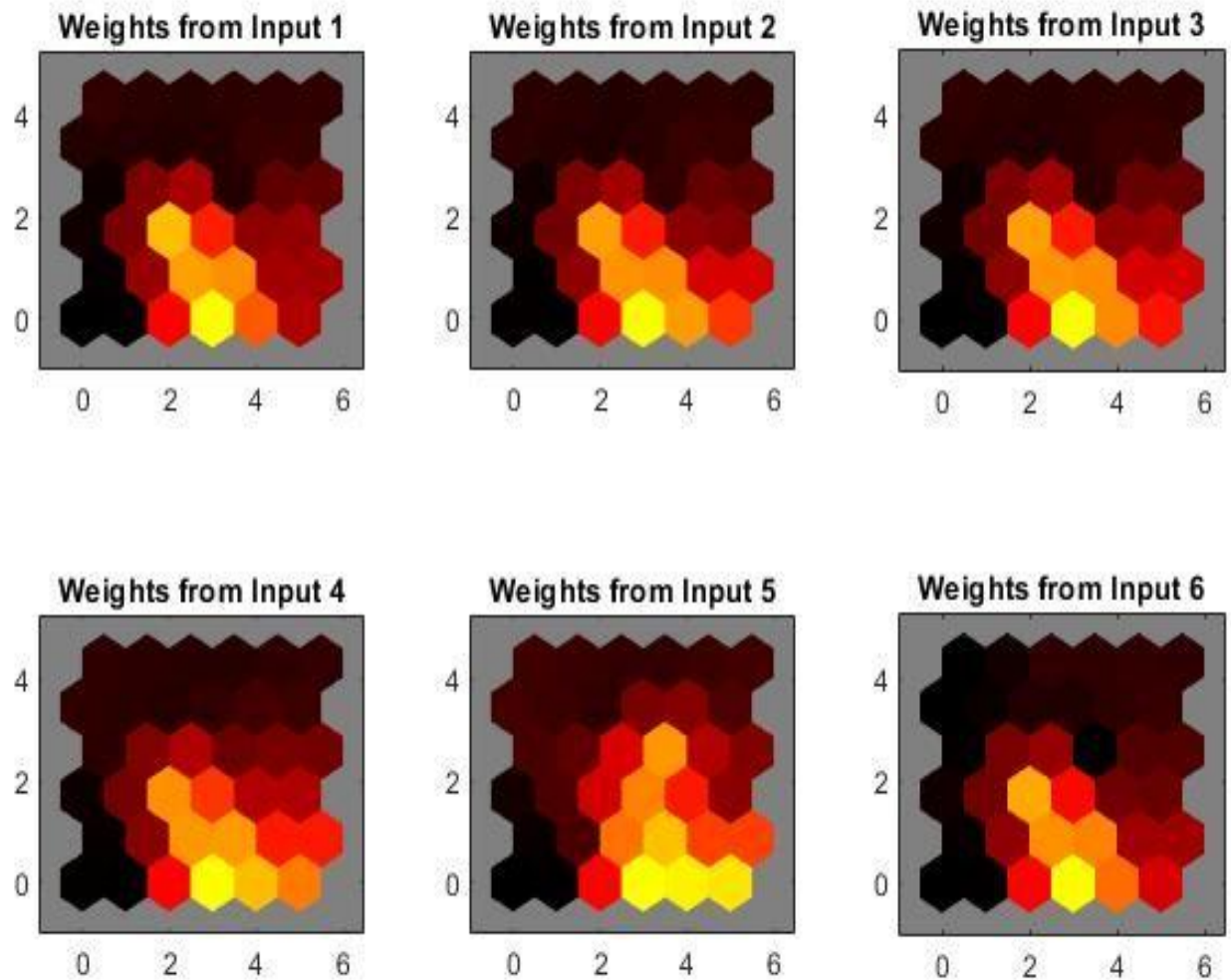

Fig. 6 Weights from input component (Input 1-fresh pulp (PP), Input 2-hot air (PH), Input 3- freeze (PF), Input 4-microwave (PMW), Input 5-microwave convective (PMWC) driedpineapple fortified rasgulla and Input6-normal rasgulla). 


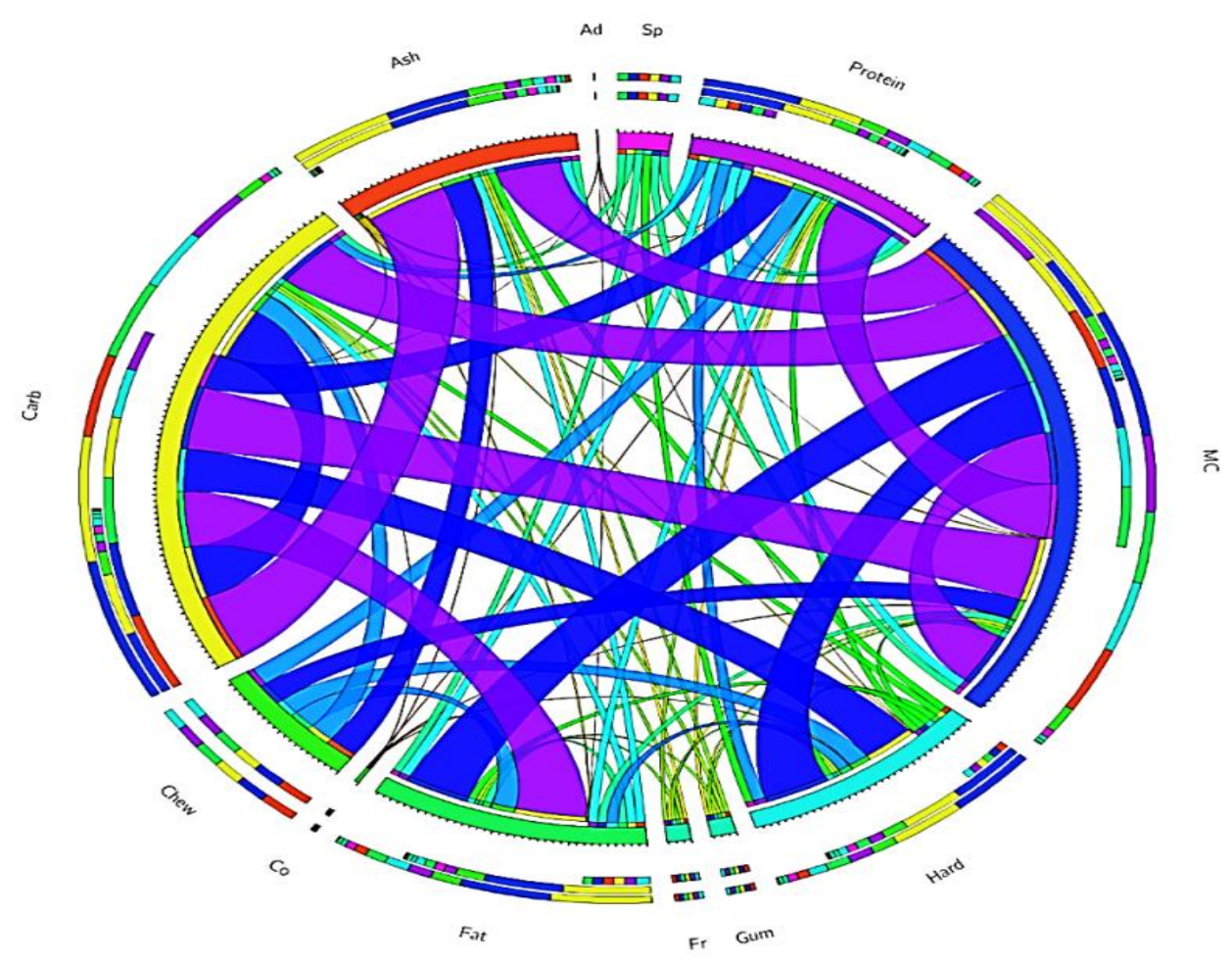

Fig. 7 Co-relation diagram of protein, fat, MC (moisture content), Carb (carbohydrate) and ash content with sensory attributes like Chew (chewiness), Co (cohesiveness), Fr (fracturability), Gum (gumminess), Hard (hardness), Ad (adhesiveness) and Sp (springiness).

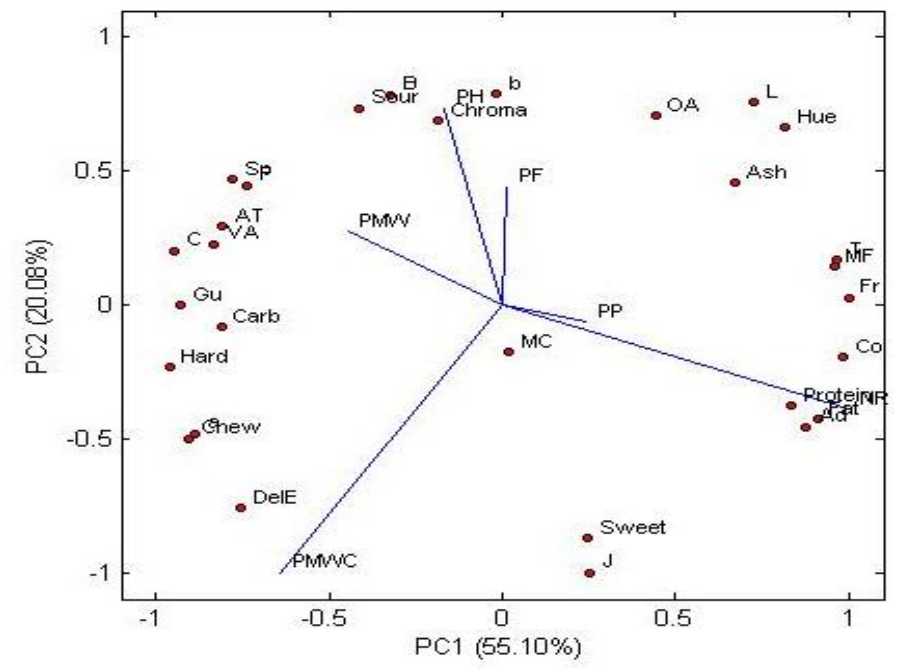

Fig. 8 Principal component analysis (PCA) bi-plot for different rasgulla and the features analysed Where, NR-Normal rasgulla, $\mathrm{PH}-\mathrm{Hot}$ air dried pineapple rasgulla, PF- Freeze dries [pineapple rasgulla, PMW-Microwave dried pineapple rasgulla, PMWCMicrowave convective dried pineapple rasgulla, springiness, gumminess, chewiness, hardness $(\mathrm{H})$, visual appearance (VA), carbohydrate, total colour difference (Del E), aftertaste, sourness, bitterness, b, chroma), overall acceptability (OA), L, hue, ash, cohesiveness, fracturability, texture $(\mathrm{T})$, protein, fat, adhesiveness, mouthfeel (MF), sweetness, juiciness, moisture content.
Also from Fig. 9 where HCA analysis with heat map showed a clear indication of six different clusters of all the observed parameters. From HCA, it is obvious that NR formed a totally different cluster, while on a broader view, the pineapple fortified rasgulla samples shared the same cluster. In the HCA model (agglomerative), the clusters were generally developed depending on the similarity or dissimilarity of the analyzed parameters. The PP and PF shared the same sub-cluster while the PH and PMW shared the other sub-cluster, pretending a similarity in the characteristics for these products. Though PMWC formed a separate sub-cluster within the purview of the cluster by all the pineapple fortified samples, this might be due to the distinctive features that appeared for the PMWC in terms of the parameters studied.

Earlier, several authors reported SOM for describing the features of food materials particularly in the field of sensory analysis. Sanchez et al., 2019 and Milovanovic et al., 2019 had studied the hydrogenation of soybean oil and the classification of different wine products based on organic acid respectively. ${ }^{[36-37,3]}$ Though it is the first kind of approach, in terms of classification by food compositional analysis, sensory attributes and physical characterization like textural and color analysis through SOM. In the SOM Fig. 5, the small blue colored hexagons are the representative of neighbor weight distance topology neurons. The red-colored lines 


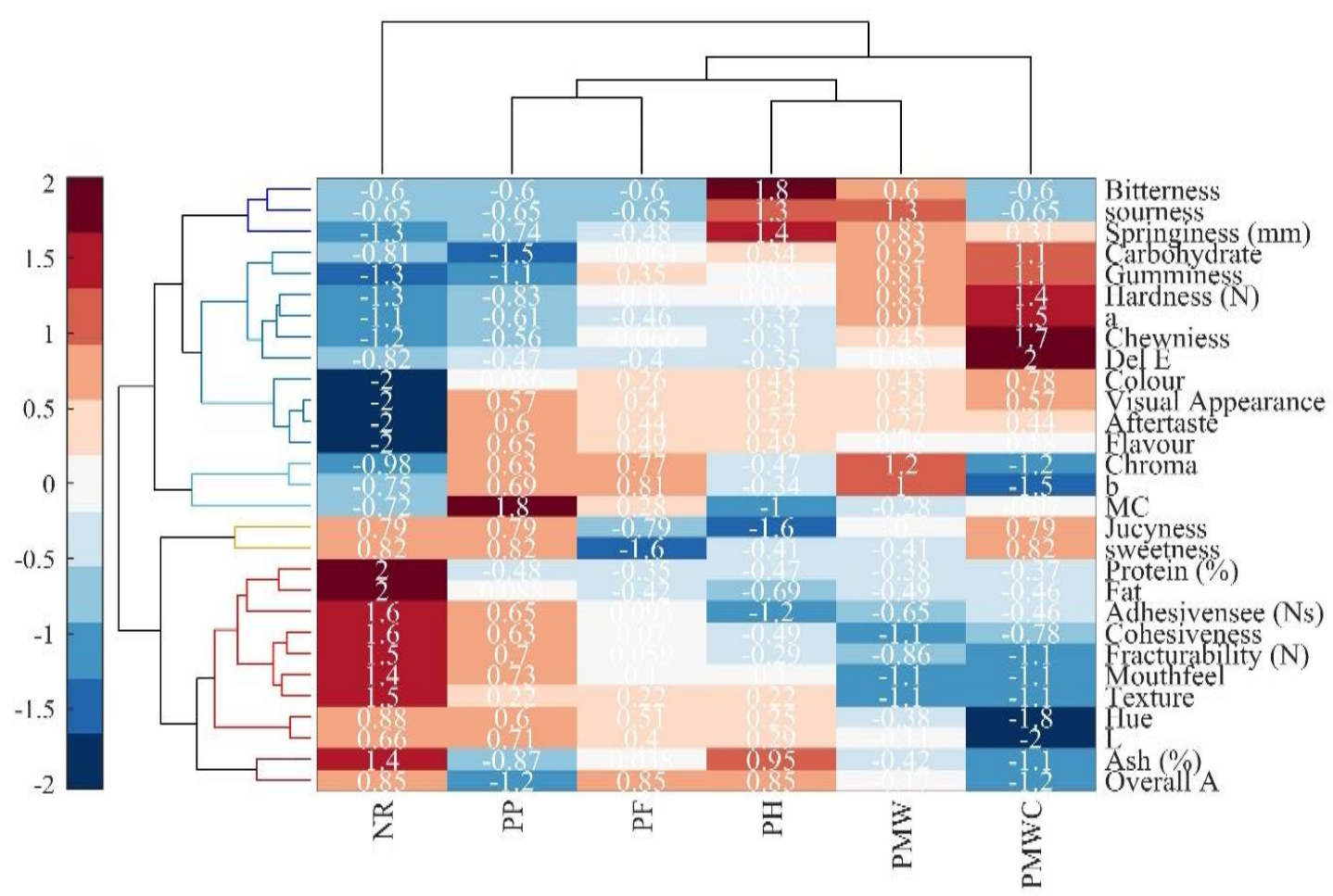

Fig. 9 Hierarchical cluster analysis (HCA) with heat map. Where, NR-Normal rasgulla, PH-Hot air dried pineapple rasgulla, PFFreeze dried [pineapple rasgulla, PMW-Microwave dried pineapple rasgulla, PMWC- Microwave convective dried pineapple rasgulla, springiness, gumminess, chewiness, hardness $(\mathrm{H})$, visual appearance (VA), carbohydrate, total colour difference (Del E), aftertaste, sourness, bitterness, b, chroma), overall acceptability (OA), L, hue, ash, cohesiveness, fracturability, texture (T), protein, fat, adhesiveness, mouthfeel (MF), sweetness, juiciness, moisture content.

generally attached adjacent neurons with a weight value. In the map, the light color represents lesser distance and the darker color indicates for larger distances in between different parameters. Both the light color and dark color indicated the clusters formed by the different parameters. From Fig. 6, a total of six light color spots were found. This indicated a total of six clusters which were formed by different parameters, and positioned nearby of each other. From HCA and PCA analysis, we have already found six major clusters formed by different parameters. Milovanovic et al., 2019, found the same result for rose, white and red wine. ${ }^{[3]}$ From Fig. 6 we can observe the weighted position. Among all the samples, it was found that PMWC was the most significant for classification in this study. Also, NR was found to significantly affect the classification pattern. Fig. 7 describes the co-relation interference of all the samples along with all the parameters.

\section{Conclusions}

From the analysis of results of all the rasgulla samples, it can be seen that fortification of pineapple in normal dairy rasgulla successfully enriched the normal rasgulla nutritionally. Higher carbohydrate $(58.03 \%)$ content was observed for PMWC while NR was found with the highest fat content $(8.01 \pm 0.79)$. $\mathrm{PH}$ possessed maximum springiness values $(5.91 \mathrm{~mm})$ and the lowest fracturability $(\mathrm{N})$ was found for PMWC $(2.51 \mathrm{~N})$. PMWC showed a maximum $\Delta \mathrm{E}$ of $48.79 \pm 3.33$ ) compared to other samples. A significantly higher IB was observed for $\mathrm{PH}$, PMW and PMWC compared to NR. PP and PMWC were ranked similarly for sensory attributes like visual appearance, overall acceptability, flavor and color. PP was found the best in terms of sensory attributes (color and flavor) among all the samples. From the XRD analysis results, it was found that there was no adequate production of sugar crystals during the process of pineapple drying that may alter the sensory quality of the fortified products. The multivariate analysis (PCA and HCA) proves how and why the pineapple fortification improves the scientific status of rasgulla processing. The PCA model elucidated that the PMW was the best sample in terms of the analyzed parameters followed by $\mathrm{PMWC}, \mathrm{PH}, \mathrm{PF}$, and PP. Similar results are obtained from all the SOM, PCA and HCA and it can be concluded that SOM may be an alternative method in the data analysis segment of food science for easier understanding of a large amount of data acquired.

\section{Supporting information}

Not applicable

\section{Conflict of interest}

There are no conflicts to declare.

\section{References}

[1] T. Sarkar, M. Salauddin, S.K. Hazra, R. Chakraborty, SN Appl. Sci., 2020, 2, 1621, doi:10.1007/s42452-020-03455-x. 
[2] T. Kohonen, Proc. IEEE, 1990, 78, 1464-1480, doi: $10.1109 / 5.58325$.

[3] M. Milovanovic, J. Žeravík, M. Obořil, M. Pelcová, K. Lacina, U. Cakar, A. Petrovic, Z. Glatz, P. Skládal, Food Chem., 2019, 284, 296-302, doi:10.1016/j.foodchem.2019.01.113.

[4] F. Marini, A.L. Magrì, R. Bucci, A.D. Magrì, Anal. Chim. Acta., 2007, 599, 232-240, doi:10.1016/j.aca.2007.08.006.

[5] J. S. Torrecilla, E. Rojo, M. Oliet, J.C. Domínguez, F. Rodríguez, J. Agric. Food Chem., 2009, 57, 2763-2769 doi:10.1021/jf803520u.

[6] E. Bona, R.S. dos Santos Ferreira da Silva, D. Borsato, D.G. Bassoli, Acta Sci. - Technol., 2012, 34, 111-119, doi: 10.4025/actascitechnol.v34i1.10892.

[7] J.V. Link, A.L. GuimarãesLemes, I. Marquetti, M.B. dos Santos Scholz, E. Bona, Food Res. Int., 2014, 59, 1-7, doi: 10.1016/j.foodres.2014.01.063.

[8] W. Melssen, R. Wehrens, L. Buydens, Chemom. Intell. Lab. Syst., 2006, 83, 99-113. 10.1016/j.chemolab.2006.02.003.

[9] T. Sarkar, M. Salauddin, S.K. Hazra, R. Chakraborty, Int. J. Intell. Networks, 2020, 1, 17-31. 10.1016/j.ijin.2020.06.001.

[10] T. Sarkar, R. Bhattacharjee, M. Salauddin, A. Giri, R. Chakraborty, Procedia Comput. Sci., Elsevier B.V., 2020, 167, 779-787, doi: 10.1016/j.procs.2020.03.410.

[11] AOAC, official methods of analysis of the association of the official analytical chemists, AOAC International 2000, Washington, DC, USA, 17th edition.

[12] T. Sarkar, M. Salauddin, S. Kumar Hazra, R. Chakraborty, Int. J. Intell. Networks, 2020, 1, 59-66, doi: 10.1016/j.ijin.2020.07.003.

[13] D. Granato, I.A. de Castro, F.V.B.W. Piekarski, C. Benincá M.L. Masson, Biol. Technol., 2011, 54, 149-159. doi: 10.1590/S1516-89132011000100020.

[14] T. Sarkar, M. Salauddin, S.K. Hazra, R. Chakraborty, J. Agric. Food Res., 2020, 2, doi: 10.1016/j.jafr.2020.100074.

[15] S. Sengupta, J. Bhowal, LWT - Food Sci. Technol., 2017, 86, 473-482. 10.1016/j.lwt.2017.08.033.

[16] U.K. Prodhan, M. Jahangir Alam, A. Sultana, M. Harun-Ar Rashid, T. Das, N. Akter, M. Mahfuzur Rahman, IOSR J. Environ. Sci., 2017, 11, 6-11, doi: 10.9790/2402-1107010611.

[17] G.M. Sun, X.M. Zhang, A. Soler, P.A. Marie- Alphonsine, Nutr. Compos. Fruit Cultiv., Elsevier Inc., 2015, 609-637, doi: 10.1016/B978-0-12-408117-8.00025-8.

[18] S. Gupta, B.S. Gowri, A.J. Lakshmi, J. Prakash, J. Food Sci. Technol., 2013, 50, 918-925, doi:10.1007/s13197-011-0407-z.

[19] S.W. Hassan, R.A. Umar, H.M. Maishanu, I.K. Matazu, U.Z. Faruk, A.A. Sani, Asian Journal of Biochemistry, 2007, 2, 349353, doi:10.3923/ajb.2007.349.353.

[20] L.E. Garcia-Amezquita, V. Tejada-Ortigoza, O.H. Campanella, J. Welti-Chanes, J. Food Qual., 2018 , 2018, doi:10.1155/2018/9105237.

[21] O.A. Ajayi, O.O. Ola, O.O. Akinwunmi, Int. Food Res. J., 2017, 24, 614-620.

[22] M. Bandyopadhyay, R. Chakraborty, U. Raychaudhuri, Int. J. Dairy Technol., 2008, 61, 290-299, doi:10.1111/j.14710307.2008.00412.x

[23] D. Ruan, H. Wang, F. Cheng, Springer Cham, 2018, 1-21. doi:10.1007/978-3-030-04777-1 1 .

[24] S. I. F. S. Martins, W. M. F. Jongen, M. A. J. S. Van Boekel, Trends Food Sci. Technol., 2000, 11, 364-373, doi: doi: 10.1016/S0924-2244(01)00022-X.

[25] N. Izli, G. Izli, O. Taskin, CYTA - J. Food., 2018, 16, 213
221, doi: 10.1080/19476337.2017.1381174.

[26] L.A. Ramallo, R.H. Mascheroni, Food Bioprod. Process, 2012, 90, 275-283, doi:10.1016/j.fbp.2011.06.001.

[27] N.D. Pham, W. Martens, M.A. Karim, M.U.H. Joardder, Food Nutr. Res., 2018, 62, doi:10.29219/fnr.v62.1292.

[28] S. Sengupta, J. Bhowal, LWT - Food Sci. Technol., 2017, 86, 473-482, doi: 10.1016/j.lwt.2017.08.033.

[29] R.S. Chavan, P.S. Prajapati, S.R. Chavan, Sens. Instrum. Food Qual. Saf., 2011, 5, 33-42, doi: 10.1007/s11694-011-91095.

[30] P. Kumar, H.N. Mishra, J. Texture Stud., 2003, 34, 249-269, doi: 10.1111/j.1745-4603.2003.tb01060.x.

[31] A.K. Adhikari, O.N. Mathur, G.R. Patil, J. Dairy Res., 1992, 59, 413-424, doi:10.1017/S0022029900030685.

[32] H.K. Desai, S. Gupta, G.R. Patil, A.A. Patel, Indian J. Dairy Sci., 1993 ,46, 123-127.

[33] K. Ten Hove, H. Das, J. Food Sci. Technol., 1995, 32, 109114.

[34] S. Sengupta, J. Bhowal, J. Microbiol. Biotechnol. Food Sci., 2017, 6, 1001-1006, doi: 10.15414/jmbfs.2017.6.4.1001-1006.

[35] S. Jaya, Int. J. Food Prop., 2009, 12, 469-481, doi: 10.1080/10942910701772071.

[36] W. A. L. Alves, S. A. de Araújo, J. H. Pessota, R. A. B. O. dos Santos, Appl. Mech. Mater., Trans Tech Publications Ltd, 2013, 2191-2194, doi:10.4028/www.scientific.net/AMM.263266.2191.

[37] J. L. Sanchez, S. B. G. Pereira, A. Tanamati, A. A. C. Tanamati, E. Bona, Emirates J. Food Agric., 2019, 31, 779-787, doi: 10.9755/ejfa.2019.v31.i10.2019.

\section{Author information}

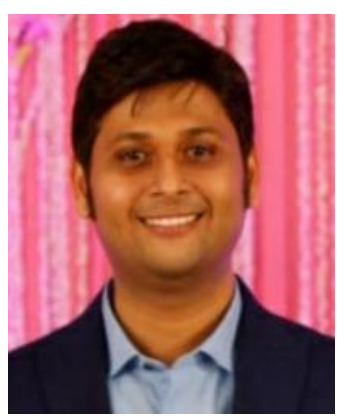

Tanmay Sarkar received his bachelor's degree in Chemistry from Calcutta University, Kolkata, India (2009), Bachelor of Technology (2012) and Master of Technology (2014) both in Food Technology and Biochemical Engineering from Jadavpur University, Kolkata, India. He has submitted his PhD thesis (2020) in the field of food process technology in Jadavpur University, Kolkata, India. He is working as a Lecturer in dept. of Food Processing Technology at Malda Polytechnic, West Bengal State Council of Technical Education, Govt. of West Bengal since 2018. His areas of interests include Optimisation, Mathematical modelling, Phytochemicals, Data science and traditional food.

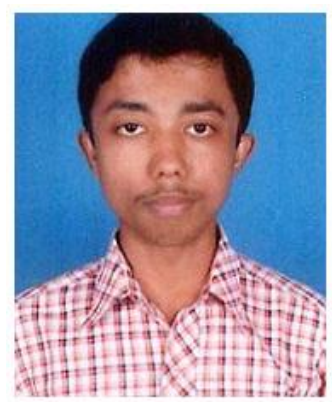

Molla Salauddin has completed his M.Tech (2020) under the supervision of Prof. Runu Chakraborty from Department of Food Technology and Biochemical Engineering, Jadavpur University. He received his B.E (Bachelor of Engineering) degree in 2018 from the Department of Food Technology 
and Biochemical Engineering, Jadavpur University. He is now working as a Food Safety officer under Kolkata Municipal Corporation, Govt. of West Bengal, India. His current research focuses on the Functional food product development and application of advance methods in the area of food engineering.

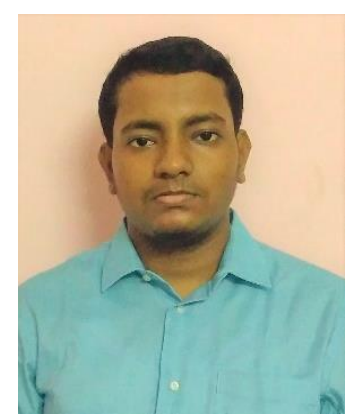

Sudipta Kumar Hazra is a final year undergraduate student in the Department of Food Technology and Biochemical Engineering at Jadavpur University since 2017. His research interests include the applications of machine learning, predictive analytics, and metaheuristic optimization in the field of food process engineering, food safety, and sensory sciences.

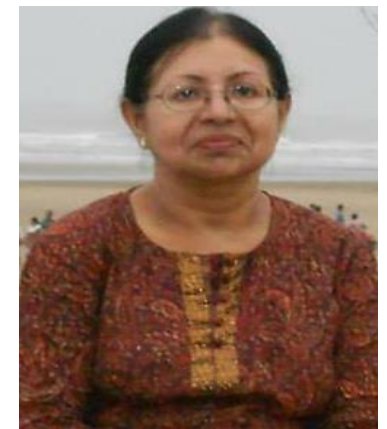

Professor Runu Chakraborty joined the department of food technology and biochemical engineering if Jadavpur University in July, 1991 as lecturer. She became reader in the year 1997 and professor in 2004. She is working in the same department till date. She has completed two terms as Head of the Department from 2001-2003 and 2015-2017. 21 students have been awarded PhD under her supervision. She has published more than 100 research papers in international and national journals and 2 patents. Her research interest is food fortification, food engineering and mathematical modelling.

Publisher's Note: Engineered Science Publisher remains neutral with regard to jurisdictional claims in published maps and institutional affiliations. 